\title{
SPHERES, SYMMETRIC PRODUCTS, AND QUOTIENT OF HYPERSPACES OF CONTINUA
}

\author{
By \\ Enrique Castañeda-Alvarado and Javier Sánchez-Martínez
}

\begin{abstract}
A continuum means a nonempty, compact and connected metric space. Given a continuum $X$, the symbols $F_{n}(X)$ and $C_{1}(X)$ denotes the hyperspace of all subsets of $X$ with at most $n$ points and the hyperspace of subcontinua of $X$, respectively. If $n>1$, we consider the quotient spaces $S F_{1}^{n}(X)=F_{n}(X) / F_{1}(X)$ and $C_{1}(X) / F_{1}(X)$ obtained by shrinking $F_{1}(X)$ to a point in $F_{n}(X)$ and $C_{1}(X)$, respectively. In this paper, we study the continua $X$ such that $S F_{1}^{n}(X)$ is homeomorphic to $C_{1}(X) / F_{1}(X)$ and we analyze when the spaces $F_{n}(X)$ and $S F_{1}^{n}(X)$ are homeomorphic to some sphere.
\end{abstract}

\section{Introduction}

A continuum means a nonempty, compact and connected metric space. The symbols $\mathbf{N}$ and $\mathbf{R}$ will denote the set of all natural numbers and real numbers, respectively. Also $I$ will be the unit interval $[0,1]$. Consider the following hyperspaces of a continuum $X$ :

$$
\begin{gathered}
2^{X}=\{A \subset X: A \text { is closed and nonempty }\}, \text { for } n \in \mathbf{N} \\
C_{n}(X)=\left\{A \in 2^{X}: A \text { has at most } n \text { components }\right\}, \\
F_{n}(X)=\left\{A \in 2^{X}: A \text { has at most } n \text { points }\right\} .
\end{gathered}
$$

These hyperspaces are considered with the Vietoris topology (see [16, Theorem 0.11 , p. 9]). The hyperspace $F_{n}(X)$ is also known as the $n^{\text {th }}$-symmetric product of $X$. Symmetric products were introduced by K. Borsuk and S. Ulam in [2], they proved that, if $n=1,2,3, F_{n}(I)$ is homeomorphic to $I^{n}$, for $n \geq 4, F_{n}(I)$ is not

2000 Mathematics Subject Classification: Primary 54B15, 54B20.

Key words and phrases: Continuum, hyperspace, quotient space, symmetric product, spheres.

Received July 7, 2013.

Revised January 6, 2014. 
homeomorphic to any subset of $\mathbf{R}^{n}$ and $F_{2}\left(S^{1}\right)$ is homeomorphic to Möbius Strip, where $S^{1}$ is the 1 -sphere. In [14], R. Molski proved that $F_{2}\left(I^{2}\right)$ is homeomorphic to the 4-cell and for $n \geq 3$ neither $F_{n}\left(I^{2}\right)$ nor $F_{2}\left(I^{n}\right)$ is homeomorphic to any subset of $\mathbf{R}^{2 n}$. In [3], R. Bott corrected Borsuk's statement (see [1]) that $F_{3}\left(S^{1}\right)$ is homeomorphic to $S^{1} \times S^{2}$ by showing that, actually $F_{3}\left(S^{1}\right)$ is homeomorphic to $S^{3}$, where $S^{n}$ denotes the $n$-sphere. In this direction, in this paper we prove the following theorem:

THEOREM 4.3. Let $X$ be a continuum. The following statements are true:

(1) (Triviality) If $n=1$, then $F_{n}(X)$ is homeomorphic to $S^{m}$ if and only if $X$ is homeomorphic to $S^{m}$,

(2) $F_{n}(X)$ is homeomorphic to $S^{m}$ for some $m \leq n$ if and only if either $n=3$ or $n=1$, and $X=S^{1}$.

Furthermore, in 1979 S. B. Nadler, Jr. introduced the hyperspace suspension of a continuum $X$ as the quotient space $C_{1}(X) / F_{1}(X),[17]$, in that paper the author studied the fixed point property of this quotient spaces. For $m, n \in \mathbf{N}$ with $m<n$ and a continuum $X$, we consider the quotient space $F_{n}(X) / F_{m}(X)$ that we will denote by $S F_{m}^{n}(X)$ obtained by shrinking $F_{m}(X)$ to a point in $F_{n}(X)$, with the quotient topology (see [6]). It is well known that $C_{1}(I) / F_{1}(I)$ and $S F_{1}^{2}(I)$ are 2-cells (see [13, In proof of Corollary 3.10, p. 129] and [6, Example 3.1]), $C_{1}\left(S^{1}\right) / F_{1}\left(S^{1}\right)$ is homeomorphic to $S^{2}$ (see [13, In proof of Corollary 3.10, p. 129]), but $S F_{1}^{2}\left(S^{1}\right)$ is the Real Projective Plane (see [6, Example 3.1]). In view of this, it is easily suspected that the spaces $X$ for which $C_{1}(X) / F_{1}(X)$ is homeomorphic to $F_{n}(X) / F_{1}(X)$ are very limited. In fact, in this paper we show the following results:

THEOREM 3.4. Let $X$ be a finite-dimensional and arcwise connected continuum. Then $C_{1}(X) / F_{1}(X)$ is homeomorphic to $S F_{1}^{2}(X)$ if and only if $X$ is homeomorphic to $[0,1]$.

THEOREM 3.6. If $Y$ is an arcwise connected continuum and $n \geq 3$, then $C_{1}(Y) / F_{1}(Y)$ is not homeomorphic to $S_{1}^{n}(X)$, for every finite dimensional continuum $X$.

Since $S F_{1}^{2}\left(S^{1}\right)$ is the Real Projective Plane (see [6, Example 3.1]), $S F_{1}^{2}\left(T_{m}\right)$ is homeomorphic to $F_{2}\left(T_{m}\right)$ (see [6, Example 3.3]) and $S F_{1}^{n}(Q)$ is homeomorphic to 
$Q$ for each $n \in \mathbf{N}$ (see [6, Example 3.1]), where $T_{m}$ is a simple $m$-od and $Q$ is the Hilbert Cube. As a consequence of the results obtained in this paper, we obtain the following

Corollary 4.8. If $X$ is a continuum and $n \geq 2$, then $S F_{1}^{n}(X)$ is not homeomorphic to $S^{m}$, for each $2 \leq m \leq n$.

Finally, the following questions remain open.

QUESTION 3.7. Can we omit the arcwise connectedness hypothesis in Theorems 3.4 and 3.6?

QUESTION 4.9. Does there exist a continuum $X$ and $n \geq 2$ such that $F_{n}(X)$ is homeomorphic to $S^{m}$ for some $m \geq 4$ ?

QUESTION 4.10. Does there exist a continuum $X$ and $m, n \geq 2$ such that $S F_{m}^{n}(X)$ is homeomorphic to $S^{m}$ for some $m \in \mathbf{N}$ ?

\section{Definitions and Preliminaries}

Given a continuum $Z$ and a subset $A$ of $Z, \operatorname{cl}_{Z}(A), \operatorname{int}_{Z}(A), \operatorname{Bd}(A)$ denotes the closure, interior and boundary of $A$ in $Z$, respectively. A subcontinuum of a space $Z$ is a continuum contained in $Z$. The symbol $|A|$ denotes the cardinality of $A$ and cone $(Z)$ denotes the quotient space $Z \times[0,1] / Z \times\{1\}$. Let $z \in Z$ and $\beta$ be a cardinal number, we say that $z$ has order less than or equal to $\beta$ in $Z$, written $\operatorname{ord}(z, Z) \leq \beta$, provided that for each open subset $U \subset Z$ such that $z \in U$, there exists $V$ an open subset of $Z$ such that $z \in V \subset U$ and $|\operatorname{Bd}(V)| \leq \beta$.

An $n$-od $(n \in \mathbf{N}$ and $n \geq 3)$ is a continuum $X$ which contains a subcontinuum $Y$ such that the complement of $Y$ in $X$ is the union of $n$ nonempty mutually separated sets (if $Y$ is a singleton and the components of $X \backslash Y$ are arcs, we say that $X$ is a simple $n$-od). A simple 3-od, will be called a simple triod. An arc is any space homeomorphic to $I$. A free $\operatorname{arc}$ in a continuum $X$ is an $\operatorname{arc} \alpha \subset X$ such that $\operatorname{int}_{X}(\alpha) \neq \varnothing$.

Given a finite collection, $U_{1}, \ldots, U_{m}$, of subsets of $X,\left\langle U_{1}, \ldots, U_{m}\right\rangle_{n}$, denote the following subset of $F_{n}(X)$

$$
\left\{A \in F_{n}(X): A \subset \bigcup_{i=1}^{m} U_{i} \text { and } A \cap U_{i} \neq \varnothing \text { for each } i=1, \ldots, m\right\} \text {. }
$$


If each $U_{i}$ is an open subset of $X$, it is known that the family of all subsets of the form $\left\langle U_{1}, \ldots, U_{m}\right\rangle_{n}$, is a basis for the topology of $F_{n}(X)$ called the Vietoris topology (see [16, Theorem 0.11, p. 9]).

Given a continuum $X, \rho_{m, n}^{X}: F_{n}(X) \rightarrow S F_{m}^{n}(X)$ denotes the natural quotient function. Also, let $F_{m}^{n}(X)$ denotes the point $\rho_{m, n}^{X}\left(F_{m}(X)\right)$.

REMARK 2.1. Using an appropriate restriction of $\rho_{m, n}^{X}$, it is clear that $S F_{m}^{n}(X) \backslash\left\{F_{m}^{n}(X)\right\}$ is homeomorphic to $F_{n}(X) \backslash F_{m}(X)$.

In this paper, dimension means inductive dimension as defined in $[16,(0.44)$, p. 21]. The symbol dim will be used to denote dimension. If $\operatorname{dim}(X) \in \mathbf{N} \cup\{-1,0\}$ we will writte $\operatorname{dim}(X)<\infty$ and $\operatorname{dim}(X)=\infty$ in other case. By [9, p. 20], for every continuum $X, \operatorname{dim}(X) \geq 1$.

The following result is a particular case of [9, Corollary 1, p. 32].

THeORem 2.2. Let $X$ be a continuum and $n \in \mathbf{N} \cup\{0\}$. If $X=Y \cup Z, Y$ is closed in $X, \operatorname{dim}(Y) \leq n$ and $\operatorname{dim}(Z) \leq n$, then $\operatorname{dim}(X) \leq n$.

COROllary 2.3. If $X$ is a continuum, $n \in \mathbf{N}, Y$ is a subcontinuum of $X$, $\operatorname{dim}(X)=n$ and $\operatorname{dim}(Y)<n$, then $\operatorname{dim}(X \backslash Y)=n$.

Proof. Is clear that $\operatorname{dim}(X \backslash Y) \leq n$. If $\operatorname{dim}(X \backslash Y)<n$, then $\operatorname{dim}(X \backslash Y) \leq$ $n-1$. By Theorem 2.2, $\operatorname{dim}(X) \leq n-1$, this is a contradiction.

\section{3. $S F_{1}^{n}(X)$ Homeomorphic to $C_{1}(X) / F_{1}(X)$}

Proposition 3.1. If $X$ is a finite-dimensional continuum, then $F_{n}(X)$ and $S F_{m}^{n}(X)$ are finite-dimensional continua.

Proof. By [8, proof of Lemma 3.1, p. 253], $\operatorname{dim}\left(F_{n}(X)\right) \leq n \cdot \operatorname{dim}(X)$, thus $F_{n}(X)$ is a finite-dimensional continuum. On the other hand, since $\operatorname{dim}\left(F_{n}(X) \backslash F_{m}(X)\right) \leq \operatorname{dim}\left(F_{n}(X)\right) \quad$ and $\quad F_{n}(X) \backslash F_{m}(X) \quad$ is homeomorphic to $S F_{m}^{n}(X) \backslash\left\{F_{m}^{n}(X)\right\}, \operatorname{dim}\left(S F_{m}^{n}(X) \backslash\left\{F_{m}^{n}(X)\right\}\right) \leq n \cdot \operatorname{dim}(X)$. Thus, by Corollary 2.3, $\operatorname{dim}\left(S F_{m}^{n}(X)\right)$ is finite.

Proposition 3.2. Let $X$ be a 1-dimensional continuum and $n \geq 2$, then $\operatorname{dim}\left(F_{n}(X)\right)=\operatorname{dim}\left(S F_{1}^{n}(X)\right)$ and $\operatorname{dim}\left(C_{1}(X)\right)=\operatorname{dim}\left(C_{1}(X) / F_{1}(X)\right)$. 
Proof. Notice that $\operatorname{dim}\left(F_{1}(X)\right)=1$. By Corollary 2.3, $\operatorname{dim}\left(F_{n}(X)\right)=$ $\operatorname{dim}\left(F_{n}(X) \backslash F_{1}(X)\right)$. We conclude

$$
\operatorname{dim}\left(S F_{1}^{n}(X) \backslash\left\{F_{1}^{n}(X)\right\}\right)=\operatorname{dim}\left(F_{n}(X)\right) .
$$

By Theorem 2.2, $\operatorname{dim}\left(S F_{1}^{n}(X)\right)=\operatorname{dim}\left(F_{n}(X)\right)$. In a similar method we can show that $\operatorname{dim}\left(C_{1}(X)\right)=\operatorname{dim}\left(C_{1}(X) / F_{1}(X)\right)$.

Lemma 3.3. If an arcwise connected continuum $X$ has hyperspace $C_{1}(X)$ of dimension at most 2, then $X$ is homeomorphic to either $S^{1}$ or $I$.

Proof. By [10, Theorem 70.1, p. 337] $X$ does not contain simple triods. Therefore, $\operatorname{ord}(x, X) \leq 2$ for every $x \in X$ because $X$ is arcwise connected. Thus, by [18, Proposition 9.5, p. 142], $X$ is an arc or $X$ is homeomorphic to $S^{1}$.

THEOREM 3.4. Let $X$ be a finite-dimensional and arcwise connected continuum. Then $C_{1}(X) / F_{1}(X)$ is homeomorphic to $S F_{1}^{2}(X)$ if and only if $X$ is homeomorphic to $[0,1]$.

Proof. If $X$ is an arc, both $C_{1}(X)$ and $S F_{1}^{2}(X)$ are 2-cells. Conversely, suppose that $C_{1}(X) / F_{1}(X)$ is homeomorphic to $S F_{1}^{2}(X)$. By Proposition 3.1, $\operatorname{dim}\left(S F_{1}^{2}(X)\right)<\infty$, thus $\operatorname{dim}\left(C_{1}(X) / F_{1}(X)\right)<\infty$. Hence, $\operatorname{dim}\left(C_{1}(X)\right)<\infty$. By [11, Theorem 2.1], we have $\operatorname{dim}(X)=1$. So, $\operatorname{dim}\left(S F_{1}^{2}(X)\right) \leq 2$ and $\operatorname{dim}\left(C_{1}(X)\right) \leq$ 2. By Lemma $3.3 X$ is an arc or $X$ is homeomorphic to $S^{1}$. But, $C_{1}\left(S^{1}\right) / F_{1}\left(S^{1}\right)$ is the 2-sphere and $S F_{1}^{2}\left(S^{1}\right)$ is homeomorphic to the real projective plane. We conclude that $X$ most be an arc.

Lemma 3.5. If $X$ is a continuum and $n \geq 3$, then $F_{n}(X)$ and $S F_{1}^{n}(X)$ does not contains 2-dimesional subsets with nonempty interior.

Proof. Suppose that there exist a 2-dimensional subset $\mathscr{D}$ of $F_{n}(X)$ with nonempty interior. Let $\mathscr{U}=\left\langle U_{1}, \ldots, U_{n}\right\rangle_{n}$ be an open subset of $F_{n}(X)$ such that $\mathscr{U} \subset \mathscr{D}$. By the denseness of $\left\{A \in F_{n}(X):|A|=n\right\}$ in $F_{n}(X)$ (see [7, In the proof of Lemma 3.1]) there is $A \in\left(F_{n}(X) \backslash F_{n-1}(X)\right) \cap \mathscr{U}$. Since $|A|=n$ we can assume that $U_{i} \cap U_{j}=\varnothing$ and $A \cap U_{i} \neq \varnothing$ for every $i, j \in\{1,2, \ldots, n\}$. Under this conditions we can take $C_{1}, C_{2}, \ldots, C_{n}$ nondegenerate subcontinua of $X$ such that $C_{i} \subset U_{i}$ for each $i$. Notice that $\left\langle C_{1}, \ldots, C_{n}\right\rangle_{n}$ is homeomorphic to $C_{1} \times \cdots \times C_{n}$. 
So, $\mathscr{U}$ contains a homeomorphic subset to $C_{1} \times \cdots \times C_{n}$. Hence $\operatorname{dim}(\mathscr{U}) \geq 3$. This is a contradiction.

THEOREM 3.6. If $Y$ is an arcwise connected continuum and $n \geq 3$, then $C_{1}(Y) / F_{1}(Y)$ is not homeomorphic to $S F_{1}^{n}(X)$, for every finite dimensional continuum $X$.

Proof. Suppose that there is a finite dimensional continuum $X$, such that $C_{1}(Y) / F_{1}(Y)$ is homeomorphic to $S F_{1}^{n}(X)$. By Proposition 3.1, $\operatorname{dim}\left(C_{1}(Y) / F_{1}(Y)\right)<\infty$. Thus, $\operatorname{dim}\left(C_{1}(Y)\right)<\infty$. By [11, Theorem 2.1], $\operatorname{dim}(Y)=1$. Let $m=\operatorname{dim}\left(C_{1}(Y)\right)$. By [10, Theorem 70.1, p. 337] and using arcwise connectedness of $Y$, this continuum does not contain simple $(m+1)$-ods. By [12, Theorem 11, p. 179], $Y$ must contain a free arc, which implies that $C_{1}(Y) / F_{1}(Y)$ contains a 2-dimensional subset with nonempty interior, but this contradicts Lemma 3.5. So, the theorem is true.

QUESTION 3.7. Can we omit the arcwise connectedness hypothesis in Theorems 3.4 and 3.6 ?

\section{Continua $X$ such that $F_{n}(X)$ and/or $S F_{1}^{n}(X)$ are $n$-spheres}

THEOREM 4.1. If $X$ is a continuum, then for each $n \geq 2$, neither $F_{n}(X)$ nor $S_{1}^{n}(X)$ is homeomorphic to $S^{2}$.

Proof. Let $X$ be a continuum such that $F_{n}(X)$ is homeomorphic to $S^{2}$ for some $n \geq 2$. Then, $F_{n}(X)$ is locally connected. By [8, Lemma 2.2, p. 252] $X$ is locally connected. Since, $\operatorname{dim}\left(S^{2}\right)=2$ then $\operatorname{dim}(X)=1$ and $n=2$. By [6, Lemma 5.9], $X$ cannot contain simple $m$-ods, for each $m \geq 3$. Therefore, by [18, Proposition 9.5, p. 142], $X$ must be an arc or a simple closed curve. But, $F_{2}(I)$ is a 2-cell and $F_{2}\left(S^{1}\right)$ is a Möbius Strip, which contradicts the assumption $F_{n}(X)$ homeomorphic to $S^{2}$.

Now, to the case $S F_{1}^{n}(X)$. Let $X$ be a continuum and suppose that $S F_{1}^{n}(X)$ is homeomorphic to $S^{2}$. Thus, $X$ is locally connected. Since $C_{1}\left(S^{1}\right) / F_{1}\left(S^{1}\right)$ is homoemorphic to $S^{2}$ (see [13, In proof of Corollary 3.10, p. 129]), by Theorem 3.6, we have $n=2$. It is clear that $\operatorname{dim}(X)$ must be equal to 1 . By [6, Example 3.3] and [6, Lemma 5.9], $X$ cannot contain simple $m$-ods, for each $m \geq 3$. So, by [18, Proposition 9.5, p. 142], $X$ is an arc or a simple closed curve. By [6, Example 3.1], in both cases $S F_{1}^{2}(X)$ is not homeomorphic to $S^{2}$. 
THEOREM 4.2. Let $X$ be a continuum. If $n \geq 2$ and $n \neq 3$, then neither $F_{n}(X)$ nor $S_{1}^{n}(X)$ is not homeomorphic to $S^{m}$, for each $2 \leq m \leq n$.

Proof. The conclusion for $n=2$ follows from Theorem 4.1.

Let $n>3$ and suppose that $F_{n}(X)$ (or $\left.S F_{1}^{n}(X)\right)$ is homeomorphic to $S^{m}$ for some $2 \leq m \leq n$. Then, $F_{n}(X)$ is locally connected. By [8, Lemma 2.2, p. 252] $X$ is locally connected. Thus, $X$ is arcwise connected. Let $\alpha$ be an arc in $X$ and $x, y \in \alpha, x \neq y$. So, there is a system of neighborhoods $\gamma$ of $\{x, y\}$ in $F_{n}(X)$ (of $\rho_{n, 1}^{X}(\{x, y\})$ in $S F_{1}^{n}(X)$, respectively) such that for every $V \in \gamma, V$ cannot be embedded in $\mathbf{R}^{n}$ (see [2]). But, each point in $S^{m}$ have a system of neighborhoods, each one of which is embedded in $\mathbf{R}^{n}$, this is a contradiction.

THEOREM 4.3. Let $X$ be a continuum. The following statements are true:

(1) (Triviality) If $n=1$, then $F_{n}(X)$ is homeomorphic to $S^{m}$ if and only if $X$ is homeomorphic to $S^{m}$,

(2) $F_{n}(X)$ is homeomorphic to $S^{m}$ for some $m \leq n$ if and only if either $n=3$ or $n=1$, and $X=S^{1}$.

Proof. (1) is true, because $F_{1}(X)$ is homeomorphic to $X$. The sufficiency of (2) is true by [3] and (1).

For the necessity of (2), suppose that $F_{n}(X)$ is homeomorphic to $S^{m}$ for some $m \leq n$. By Theorem 4.2, $n=1$ or $n=3$. If $n=1$, since $F_{1}(X)$ is homeomorphic to $X$, then $X$ is homeomorphic to $S^{1}$. If $n=3$, by [5, Corollary 5.9], $X$ is homeomorphic to $S^{1}$.

Since each continuum $Z$ is a compact, metric space, cone $(Z)$ is homeomorphic to the so-called geometric cone over $Z$ (see [18, Exercise 3.28, p. 47]). So, the following remark is easy to be seen.

REMARK 4.4. If $Z$ is a continuum and $n \geq 2$, cone $(Z)$ can be embedded in $\mathbf{R}^{n}$ if and only if $Z$ can be embedded in $\mathbf{R}^{n-1}$.

Lemma 4.5. If $T_{3}$ is a simple triod, then $F_{3}\left(T_{3}\right)$ and $S F_{1}^{3}\left(T_{3}\right)$ can not be embedded in $\mathbf{R}^{3}$.

Proof. Let $v_{1}, v_{2}$ and $v_{3}$ the end points of $T_{3}$. Let

$$
Z=\left\{A \in F_{3}\left(T_{3}\right): A \cap\left\{v_{1}, v_{2}, v_{3}\right\} \neq \varnothing\right\} .
$$


Since cone $(Z)$ is homeomorphic to $F_{3}\left(T_{3}\right)$ (see [4]). In order to prove that $F_{3}\left(T_{3}\right)$ can not be embedded in $\mathbf{R}^{3}$ we only need to show $Z$ can not be embedded in $\mathbf{R}^{2}$. Let $v$ be the vertex of $T_{3}$. Is easy to construct a system of neighborhoods $\gamma$ of the point $\left\{v_{1}, v\right\}$ such that for each $V \in \gamma, V$ contain a homeomorphic copy of $T_{3} \times I$, but by [4, Lemma 3.1, p. 58], each one of them can not be embedded in $\mathbf{R}^{2}$. In a similar method, we can show that $S F_{1}^{3}\left(T_{3}\right)$ can not be embedded in $\mathbf{R}^{3}$.

Lemma 4.6. If $X$ is homeomorphic to I or $S^{1}$, then $F_{3}(X)$ is not homeomorhic to $S F_{1}^{3}(X)$.

Proof. First suppose that $X$ is homeomorphic to $I$. By [2, Theorem 6, p. 880], there exists a homeomorphism $k: F_{3}(X) \rightarrow D$ where

$$
D=\left\{(x, y, z) \in \mathbf{R}^{3}: x^{2}+y^{2}+z^{2} \leq 1\right\}
$$

and $k\left(F_{1}(X)\right)$ is the linear segment that joint the points $(0,0,1)$ and $(0,0,-1)$. So, $S F_{1}^{3}(X)$ is not homeomorphic to $I^{3}$, and then $F_{3}(X)$ and $S F_{1}^{3}(X)$ are not homeomorphics.

Now, if $X$ homeomorphic to $S^{1}$, suppose that there is a homeomorphism $h: S F_{1}^{3}(X) \rightarrow F_{3}(X)$. Let $p=h\left(F_{1}^{3}(X)\right)$. By Remark 2.1, $S^{3} \backslash\{p\}$ is homeomorphic to $F_{3}(X) \backslash F_{1}(X)$. On the other hand, $S^{3} \backslash\{p\}$ is homeomorphic to $\mathbf{R}^{3}$. Moreover by [15, Theorem 2] there is a homeomorphism between $F_{3}(X)$ and $S^{3}$ such that the image of $F_{1}(X)$ is a trefoil knot $T$ in $S^{3}$. Thus, $\mathbf{R}^{3}$ and $S^{3} \backslash T$ are homeomorphic. But, its first fundamental groups $\pi_{1}\left(\mathbf{R}^{3}\right)$ and $\pi_{1}\left(S^{3} \backslash T\right)$ are not isomorphic, which is a contradiction.

THEOREM 4.7. If $X$ is a continuum, then $S F_{1}^{3}(X)$ is not homeomorphic to $S^{3}$.

Proof. Suppose that $X$ is a continuum and $S F_{1}^{3}(X)$ is homeomorphic to $S^{3}$. So, $X$ is locally connected. By Lemma $4.5, X$ cannot contain simple triods, because each point in $S^{3}$ has a system of neighborhoods, $\gamma$, such that for each $V \in \gamma, V$ can be embedded in $\mathbf{R}^{3}$. So, $X$ must be an arc or a simple closed curve. This contradicts Lemma 4.6.

By Theorems 4.2 and 4.7 we obtain the following corollary.

COROLlaRY 4.8. If $X$ is a continuum and $n \geq 2$, then $\operatorname{SF}_{1}^{n}(X)$ is not homeomorphic to $S^{m}$, for each $2 \leq m \leq n$. 
To finish this paper, we pose the following questions.

QUESTION 4.9. Does there exist a continuum $X$ and $n \geq 2$ such that $F_{n}(X)$ is homeomorphic to $S^{m}$ for some $m \geq 4$ ?

QUESTION 4.10. Does there exist a continuum $X$ and $m, n \geq 2$ such that $S F_{m}^{n}(X)$ is homeomorphic to $S^{m}$ for some $m \in \mathbf{N}$ ?

\section{Acknowledgment}

The authors thank the referee's suggestion, which helped to substantially improve the writing of this paper.

\section{References}

[1] Borsuk, K., On the third symmetric potency on the circumference, Fund. Math., 36 (1949), $235-244$.

[2 ] Borsuk, K. and Ulam, S., On symmetric products of topological spaces, Bull. Amer. Math. Soc., 37 (1931), 875-882.

[ 3 ] Bott, R., On the third symmetric potency of $S_{1}$, Fund. Math., 39 (1952), 364-368.

[4] Castañeda, E., Symmetric products as cones and products, Topology Proc., 28 (2004), $55-67$.

[5] Castañeda-Alvarado, E. and Illanes, A., Finite graphs have unique symmetric products, Topology Appl., 153 (2006), 1434-1450.

[6] Castañeda-Alvarado, E. and Sánchez-Martínez, J., On the unicoherence of $F_{n}(X)$ and $S F_{m}^{n}(X)$ of continua, Topology Proc., 42 (2013), 309-326.

[ 7 ] Castañeda-Alvarado, E., Orozco-Zitli, F. and Sánchez-Martínez, J., Induced mappings between quotient spaces of symmetric products of continua, Topology Appl., 163 (2014), 66-76.

[8] Curtis, D. and Nhu, N. T., Hyperspaces of finite subsets which are homeomorphic to $\aleph_{0}$-dimensional linear metric spaces, Topology Appl., 19 (1985), 251-260.

[9] Hurewicz, W. and Wallman, H., Dimension Theory, Princeton, 1948.

[10] Illanes, A. and Nadler, S. B., Jr., Hyperspaces, Fundamentals and recent advances, Monographs and Textbooks in Pure and Applied Mathematics, 216, New York: Marcel Dekker, Inc., 1999.

[11] Levin, M. and Sternfelf, Y., The space of subcontinua of a 2-dimensional continuum is infinitely dimensional, Proc. Amer. Math. Soc., 125 (1997), 2771-2775.

[12] Macías, S., On symmetric products of continua, Topology Appl., 92 (1999), 173-182.

[13] Macías, S., On the $n$-fold hyperspace suspension of continua, Topology Appl., 138 (2004), $125-138$.

[14] Molski, R., On symmetric products, Fund. Math., 44 (1957), 165-170.

[15] Mostovoy, J., Lattices in C and finite subsets of a circle, Am. Math. Mon. 111 (2004), 357-360.

[16] Nadler, S. B., Jr., Hyperspaces of Sets, Monographs and Textbooks in Pure and Applied Mathematics, 49, New York: Marcel Dekker, Inc., 1978.

[17] Nadler, S. B., Jr., A fixed point theorem for hyperspace suspension, Houston J. Math., 5 (1979), 125-132.

[18] Nadler, S. B., Jr., Continuum Theory. An Introduction, Monographs and Textbooks in Pure and Applied Mathematics, 158. New York: Marcel Dekker, Inc., 1992. 
84 Enrique Castañeda-Alvarado and Javier SÁnchez-Martínez

Universidad Autónoma del Estado de México

Facultad de Ciencias

Instituto Literario No. 100, Col. Centro, C.P. 50000, Toluca

Estado de México, México

E-mail: eca@uaemex.mx

E-mail: jsanchezm@uaemex.mx 